\title{
Sensibilidade de diferentes sementes em ensaio de fitotoxicidade
}

\section{Sensitivity of different seeds in phytotoxicity assay}

\author{
Thais de Araujo Goya Peduto; ; Tatiane Araújo de Jesus ${ }^{2}$; Marcio Yukihiro Kohatsu ${ }^{3}$ \\ ${ }^{1}$ Aluna do Curso de Pós-graduação em Ciência e Tecnologia Ambiental, Universidade Federal do ABC, Santo André, São \\ Paulo, Brasil. Orcid: https://orcid.org/0000-0001-9872-5904. E-mail: thais.goya@outlook.com \\ ${ }^{2}$ Professora no Programa de Mestrado Acadêmico em Ciência e Tecnologia Ambiental, Universidade Federal do ABC, \\ Santo André, São Paulo, Brasil. Orcid: https://orcid.org/0000-0002-5206-6584. E-mail: tatiane.jesus@ufabc.edu.br \\ ${ }^{3}$ Aluno no Curso de Pós-graduação em Ciência e Tecnologia Ambiental, Universidade Federal ABC, Santo André, São \\ Paulo, Brasil. Orcid: https://orcid.org/0000-0003-2998-8780. E-mail: mykohatsu@gmail.com
}

RESUMO: A Ecotoxicologia estuda a contaminação ambiental e os efeitos das substâncias químicas em seres vivos, investigando se são passíveis de causar danos aos organismos, levando em conta a sensibilidade à exposição por agentes tóxicos. Os testes de toxicidade com organismos vegetais possuem vantagens como: a simplicidade na realização do ensaio, sensibilidade na indicação de substâncias tóxicas e baixo custo. Como limitações, estas análises não permitem identificar especificamente os contaminantes que causam a toxicidade. Dessa forma, visou-se neste estudo analisar e comparar os parâmetros macroscópicos que demonstram alterações no crescimento inicial de seis espécies vegetais fortemente empregadas como bioindicadoras de toxicidade e analisar o índice de germinação. Eles consistiram em incubar $(n=10)$ sementes das espécies Allium cepa (Cebola), Cucumis sativus (Pepino), Lepidium sativum (Agrião do jardim), Lactuca sativa (Alface), Sinapis alba (Mostarda) e Eruca sativa (Rúcula), por diferentes períodos, em contato com três soluções de controle (Dicromato de Potássio, água ISO e água destilada) à temperatura de $20^{\circ} \mathrm{C}$. Foi realizado também o comparativo econômico de aquisição das sementes no comércio. Os ensaios de germinação apresentaram diferentes respostas, concluindo que a semente da espécie $S$. alba obteve o melhor desempenho no teste de fitotoxicidade, por apresentar alta germinação em menor tempo de incubação, facilidade de avaliação das plântulas, elevado grau de sensibilidade frente aos compostos estimulante e inibidor e menor valor de compra.

Palavras-chave: Bioindicadores vegetais; Ecotoxicologia; Ensaio de germinação.

ABSTRACT: Ecotoxicology studies the environmental contamination and the effects of chemicals on living beings, investigating if they are likely to cause damage to organisms, taking into account the sensitivity to exposure to toxic agents. Toxicity tests with plant organisms have advantages such as simplicity in carrying out the test, sensitivity in the indication of toxic substances and low cost. As limitations, these analyzes do not allow to identify specifically the contaminants that cause toxicity. Thus, the aim of this study was to analyze and compare macroscopic parameters that demonstrate changes in the initial growth of six plant species strongly used as toxicity bioindicators and to analyze the germination index. They consisted of incubating $(n=10)$ seeds of Allium cepa (Onion), Cucumis sativus (Cucumber), Lepidium sativum (Garden Cress), Lactuca sativa (Lettuce), Sinapis alba (Mustard) and Eruca sativa (Arugula) species, for different periods, in contact with three control solutions (Potassium Dichromate, ISO water and distilled water) at a temperature of $20{ }^{\circ} \mathrm{C}$. The economic comparison of seed acquisition in the trade was also performed. The germination tests showed different responses, concluding that the seed of the species $S$. alba obtained the best performance in the phytotoxicity test, because it presented high germination in a shorter incubation time, ease of seedling evaluation, high sensitivity to the stimulant compounds and inhibitor and lower purchase value.

Keywords: Ecotoxicology; Germination teste; Vegetable bioindicators.

DOI: $10.18554 /$ rbcti.v4i2.3698 
INTRODUÇÃO

A Ecotoxicologia visa prever e entender os efeitos das substâncias químicas em seres vivos e comunidades naturais, e investigar se as substâncias naturais e contaminantes são passíveis de causar danos aos organismos (SILVA; MATTIOLO, 2011). Internacionalmente, há órgãos que normatizam testes ecotoxicológicos, como: International Standardization Organization (ISO), United States Environmental Protection Agency (USEPA), Environmental Canada ou American Society for Testing Materials (ASTM) (MATSUBARA, 2018). Nacionalmente, a nível federal, resoluções como CONAMA - Conselho Nacional do Meio Ambiente n. 357/05, complementada pela 430/11 (BRASIL, 2005) e CONAMA n. 344/04 (BRASIL, 2004) instituem testes ecotoxicológicos como parâmetro de qualidade das águas e efluentes e para a disposição de sedimento dragado, respectivamente. A CETESB - Companhia Ambiental do Estado de São Paulo, solicita esses testes para processos de licenciamento, caracterização e acompanhamento de impactos de novos empreendimentos, avaliação de risco de áreas contaminadas, entre outros e indica a utilização dos métodos normatizados pela ABNT - Associação Brasileira de Normas Técnicas para os organismos aquáticos.

Entretanto, testes com espécies vegetais que visam determinar a inibição da germinação e o crescimento das radículas das sementes não são considerados como um dos parâmetros de análise da toxicidade de amostras de água, efluentes ou sedimentos pela ABNT (ABNT, 2016) e CETESB (CETESB, 2017), abrindo lacuna para estudos das sementes como bioindicadoras de toxicidade. Os testes propostos com organismos vegetais possuem vantagens, tais como: a simplicidade na realização do ensaio, baixo custo, baixa manutenção, sensibilidade na indicação qualitativa da presença de substâncias tóxicas ou inibidores biológicos, método reprodutível, não requer grandes equipamentos, ausência de sazonalidade, fácil aquisição das sementes no comércio, pequeno volume de amostra e rápida germinação (PELEGRINI et al., 2006; RUBINGER, 2009; FRECCIA, 2011; ROCHA, 2017). Como limitações, estas análises não permitem identificar quais são os contaminantes que causam a toxicidade especificamente, e o fato de não existir um método universalizando os testes de fitotoxicidade para o composto analisado origina em conclusões diferentes dependendo do momento da avaliação (BELO, 2011).

O teste de germinação é padronizado pelo Ministério da Agricultura, Pecuária e Abastecimento desde 1967 com o documento intitulado "RAS" - Regras para Análise de Sementes, em que visa determinar o potencial máximo de germinação a fim de comparar a qualidade de lotes de sementes e estimar o valor para plantio (BRASIL, 2009). O "RAS" ressalta que a realização dos testes de germinação em campo pode não ser satisfatória, devido à falta de domínio sobre fatores externos, como temperatura e exposição à luz, e padroniza estas condições visando a reprodutibilidade do teste (BRASIL, 2009).

Já o teste de fitotoxicidade, com o auxílio do teste de germinação, visa avaliar os efeitos das toxinas presentes no meio de cultivo de plantas, identificando águas e solos contaminados através do índice de germinação - IG, podendo variar entre teste crônico e agudo, em que sua principal diferença é o tempo que o bioindicador permanece exposto ao potencial contaminante (DEVESA-REY et al., 2008). De acordo com Carniato et al. (2007), Costa (2010) e Lapa et al. (2014), os testes crônicos são caracterizados por permanecerem um longo período, em parte ou abrangendo todo o ciclo de vida do organismo-teste, exposto ao potencial contaminante tóxico. Os testes agudos são realizados em laboratórios, onde as condições são específicas e controladas, reduzindo a 
interferência das variáveis climáticas presentes em campo. Nos primeiros dias de desenvolvimento das plântulas ocorrem diversos processos fisiológicos, os quais podem ser comprometidos pela presença de toxinas. Os bioensaios partem desse princípio, onde os parâmetros de análise são: o crescimento da radícula e do hipocótilo, indicando a capacidade da plântula de se estabelecer e se desenvolver, o que influencia diretamente na germinação (ROCHA, 2017).

O uso de organismos testes tem sido bastante variado em termos de metodologias e finalidades ao longo dos anos. Nos estudos em que foram realizados ensaios fitotoxicológicos, pode-se citar o emprego de diversas espécies entre flores, cereais e hortaliças, como: Anthemis marítima, Armeria marítima, Eruca sativa, Hordeum vulgare, Lactuca sativa, Lepidium sativum L., Lolium multiflorum, Lolium perene, Medicago sativa L., Phaseolus radiatus L., Plantago coronopus, Raphanus sativus L., Sinapis alba e Triticum aestivum (FANTIN et al., 2009; YERUSHALMI et al., 2010; HIMANEN et al., 2012; BEDELL et al., 2014a; BEDELL et al., 2014b; EMAMI et al., 2014; MACÍA et al., 2014; KUNDRÁT et al., 2017; SONG et al., 2017; ZHANG et al., 2017; KOHATSU et al., 2018). Para a realização dos ensaios fitotóxicos, adota-se o controle que é uma solução que causa efeitos previsíveis sobre os organismos indicadores.

Silva e Mattiolo (2011) analisaram diferentes concentrações do dicromato de potássio em sementes da espécie Lactuca sativa, concluindo que não houve dano letal na germinação das sementes, entretanto apresentou efeito fitotóxico quanto ao crescimento da radícula.

A água ISO é normatizada pela Organização para Cooperação e Desenvolvimento Econômico (OECD, 2004) em que visa promover a germinação a partir da disponibilidade de nutrientes. Como exemplo, esta solução foi aplicada no estudo da fitotoxicidade de águas superficiais na Região Metropolitana de São Paulo por Kohatsu et al. (2018). Os autores obtiveram êxito na classificação de toxicidade, apontando fitotoxicidade moderada para o Córrego Ribeirão Pires e não fitotoxicidade para uma estação de região mais preservada do reservatório Rio Grande, um braço da Represa Billings. A água destilada, por sua vez, é carente de íons, compostos orgânicos e inorgânicos, sendo formada apenas pela ligação dos átomos de hidrogênio e oxigênio. Belo (2011) adotou água destilada a fim de avaliar os efeitos dos extratos aquosos de materiais da compostagem em bioensaios com sementes de agrião de jardim (Lepidium sativum).

Nesse contexto, o presente estudo teve por objetivo avaliar e comparar a sensibilidade de diferentes sementes em ensaio de fitotoxicidade.

\section{MÉTODO}

As sementes foram compradas em diferentes lojas, sendo comparados os valores de compra por massa de sementes, com exclusão da variável de preço promocional.

Os bioensaios consistiram em incubar as sementes a $20^{\circ} \mathrm{C}$ por diferentes períodos as sementes em contato com uma solução e avaliar os parâmetros macroscópicos de crescimento através da medição do comprimento das radículas. Os ensaios foram conduzidos no Laboratório de Caracterização de Matrizes Ambientais da Universidade Federal do ABC. Sementes comerciais, isentas de agrotóxicos, tratamento ou peletização, em suas respectivas marcas e lotes foram adquiridas: Isla ${ }^{2}$, Lote: 122046 - Allium cepa (Cebola Baia Super Precoce); Isla $囚$, Lote: 118953 - Cucumis sativus (Pepino); Isla®, Lote: 120100 - Lepidium sativum (Agrião do jardim); Isla ${ }^{\circledR}$ Lote: 118943 - Lactuca sativa (Alface Simpson sementes pretas); sem marca e sem lote - Sinapis alba (Mostarda); Isla ${ }^{\circledR}$, e, Lote: 
120285 - Eruca sativa (Rúcula).

Para o controle positivo adotou-se o uso da água ISO (OECD, 2004). Para o controle negativo, solução de dicromato de potássio à concentração de $50 \mathrm{mg} \mathrm{L}^{-1}$ (SILVA; MATTIOLO, 2011). Adotou-se água destilada como controle neutro (BELO, 2011). O preparo da água ISO seguiu a metodologia descrita em OECD (2004), diluindo cloreto de potássio $\left(1,176 \mathrm{mg} \mathrm{L}^{-1}\right)$, sulfato de magnésio $\left(0,493 \mathrm{mg} \mathrm{L}^{-1}\right)$, bicarbonato de sódio $(0,259$ $\left.\mathrm{mg} \mathrm{L}^{-1}\right)$ e cloreto de potássio $\left(0,023 \mathrm{mg} \mathrm{L}^{-1}\right)$ em água destilada sob agitação até a diluição total das substâncias, válida por 3 meses após o preparo. A escolha da concentração de $50 \mathrm{mg} \mathrm{L}^{-1}$ de dicromato de potássio ocorreu através do estudo de Silva e Mattiolo (2011), onde os autores estudaram diferentes concentrações e concluíram que atuou no metabolismo da semente e inibiu o seu crescimento.

Os testes foram realizados seguindo a metodologia adotada por Kohatsu et al. (2018) com adaptação no número de sementes a serem testadas. Foram utilizadas placas de Petri de vidro (90 $\mathrm{mm}$ de diâmetro) devidamente desinfestadas com ácido clorídrico $\left(\mathrm{HCl} 10 \% \mathrm{v} / \mathrm{v}\right.$ ), cobertas com papel filtro qualitativo (marca Unifil ${ }^{\circledR}$ ), gramatura de $80 \mathrm{~g} \mathrm{~m}^{-2}$, com diâmetro de $90 \mathrm{~mm}$. Em cada placa foram adicionados $3 \mathrm{~mL}$ da solução a ser testada e 10 sementes dispostas em linha reta a $3 / 4$ da altura placa (Figura 1). Foram montadas quatro réplicas para cada solução. As placas, devidamente identificadas e embaladas com filme PVC, foram colocadas em incubadora (Solab Científica ${ }^{\circledR}$, tipo BOD), inclinadas a $45^{\circ}$.

Figura 1. Imagem ilustrativa do ensaio de fitotoxicidade com sementes.

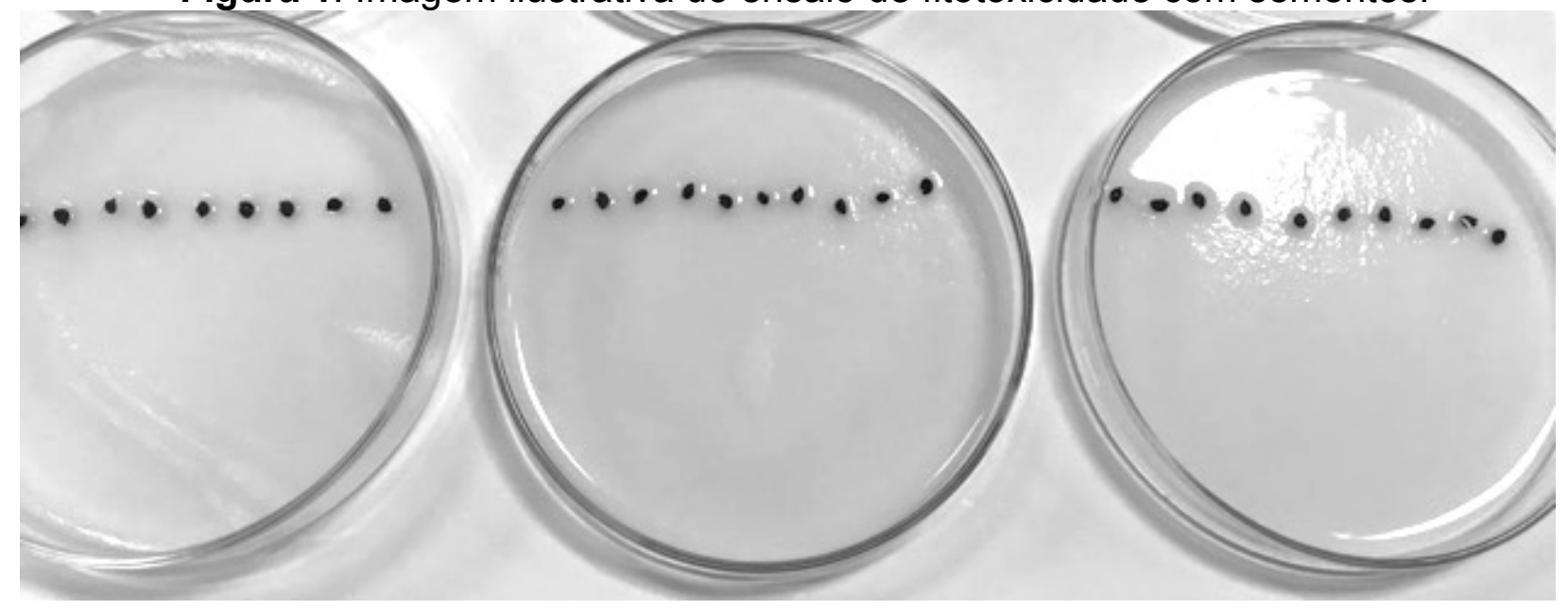

As instruções para realização dos testes de germinação, de acordo com o RAS, para as espécies propostas, estão descritos na Tabela 1. Ainda de acordo com o RAS, os tempos de incubação adotados no trabalho correspondem ao tempo de germinação para realização da primeira contagem, chamados de teste de vigor, pois apenas as sementes vigorosas germinam no período inicial de incubação (BRASIL, 2009).

Baseado em testes de fitotoxicidade realizados na literatura adotaram-se as seguintes referências para o período de incubação das sementes: para a espécie $A$. cepa, o período de germinação foi de 168 horas (MACAN, et al., 2012), C. sativus e L. sativum, 96 horas (GUERRA; ANGELIS, 2012), S. alba, 72 horas (KOHATSU et al., 2018), L. sativa e E. sativa, 120 horas (FANTIN, et al., 2009; MACENA et al., 2017), todos à temperatura de $20^{\circ} \mathrm{C} \pm 2{ }^{\circ} \mathrm{C}$, respeitando o período de incubação para os testes de germinação (entre a contagem inicial e final), a temperatura $\left(20-30^{\circ} \mathrm{C}\right)$ e o substrato (sobre papel) proposto pelas RAS (BRASIL, 2009), todos na ausência de luz. 
Tabela 1. Instruções para realização dos testes de germinação de sementes. Legenda: (EA) Entre Areia; (EP) Entre Papel; (RP) Rolo de Papel; (SA) Sobre Areia; (SP) Sobre Papel.

\begin{tabular}{ccccccc}
\hline \multicolumn{2}{c}{ SEMENTE } & \multicolumn{2}{c}{ INCUBAÇÃO (dias) } & & \\
\cline { 1 - 3 } Nome popular & Nome científico & Inicial & Final & & \\
\cline { 1 - 3 } Cebola & Allium cepa & 6 & 12 & $20 ; 15$ & EA; EP; SP \\
Pepino & Cucumis sativus & 4 & 8 & $20-30 ; 25$ & EA; RP; SP \\
$\begin{array}{c}\text { Agrião do } \\
\text { Jardim }\end{array}$ & $\begin{array}{c}\text { Lepidium } \\
\text { sativum }\end{array}$ & 4 & 10 & $20-30 ; 20 ; 15$ & AS; SP \\
Alface & Lactuca sativa & 4 & 7 & $20 ; 15$ & EP; AS; SP \\
Mostarda & Sinapis alba & 3 & 7 & $20-30 ; 20$ & AS; SP \\
Rúcula & Eruca sativa & 4 & 7 & 20 & EP; SP \\
\hline
\end{tabular}

Fonte: Adaptado de Brasil, 2009.

Após a incubação, as sementes foram analisadas de acordo com duas variáveis: quantidade de sementes germinadas e comprimento da raiz, possibilitando o cálculo do Índice de Germinação (IG). Para medir o comprimento das raízes foi utilizado um paquímetro digital de $150 \mathrm{~mm}$ com precisão de 0,001 cm (Marca Matrix®, Modelo 316119 MFB). A porcentagem de germinação absoluta (\%GA) foi obtida através da Equação 1, a porcentagem do crescimento relativo das radículas (\%CRR) e o índice de germinação (IG) nas equações 2 e 3, respectivamente, baseado nos estudos de Silva e Mattiolo (2011); Matsubara (2018) e Kohatsu et al. (2018). Para a classificação de Lumbaque et al. (2016), foi utilizada a equação 4 , que consiste no crescimento relativo da radícula (CRR).

$\% \mathrm{GA}=\frac{\text { Média aritmética das sementes germinadas no controle (positivo ou negativo) }}{\text { Média aritmética das sementes germinadas no controle neutro }} \times 100$

$\% \mathrm{CRR}=\frac{\text { Média aritmética do comprimento das radículas do controle (positivo ou negativo) }}{\text { Média aritmética do comprimento das radículas no controle neutro }} \times 100$

$\mathrm{IG}=\frac{(\% \mathrm{GA}) \times(\% \mathrm{CRR})}{100}$

$\mathrm{CRR}=\frac{\text { Média aritmética do comprimento das radículas do controle (positivo ou negativo) }}{\text { Média aritmética do comprimento das radículas no controle neutro }}$

A classificação que determina o grau de toxicidade presente na amostra pode ser feita através do Crescimento Relativo da Radícula (CRR) e do Índice Germinação (IG) seguindo as metodologias de Lumbaque et al. (2016) e Belo (2011), cujas classificações são apresentadas nas Tabelas 2 e 3. 
Tabela 2. Classificação a partir do Crescimento Relativo da Radícula (CRR).

\begin{tabular}{cc} 
FAIXA & EFEITO \\
\hline $0<\mathrm{CRR}<0,8$ & Inibição no crescimento da raiz \\
$0,8 \leq \mathrm{CRR} \leq 1,2$ & Nenhum efeito significativo \\
$\mathrm{CRR}>1,2$ & Estímulo do crescimento da raiz \\
\hline
\end{tabular}

Fonte: Lumbaque et al., 2016.

Tabela 3. Classificação a partir do Índice de Germinação (IG).

\begin{tabular}{cc}
\hline ÍNDICE DE GERMINAÇÃO (IG) & CLASSIFICAÇÃO \\
\hline$<30$ & Muito fitotóxico \\
$30-60$ & Fitotóxico \\
$60-80$ & Moderadamente fitotóxico \\
$80-100$ & Não fitotóxico \\
$>100$ & Potencializa a germinação \\
\hline
\end{tabular}

Fonte: Belo, 2011.

\section{Tratamentos e análise}

Para analisar estatisticamente os resultados experimentais obtidos em laboratório recorreu-se primeiramente ao teste de normalidade Shapiro-Wilk, e, posteriormente, à análise de variância (ANOVA) de fator único e o teste de Tukey, realizado no software Past para a variável do crescimento radicular e do IG.

\section{RESULTADOS E DISCUSSÃO}

As sementes da espécie $A$. cepa não germinaram devido à ausência de tratamento recomendado nas RAS (BRASIL, 2009), em que se indica que as sementes sejam resfriadas em baixa temperatura $\left(5-10{ }^{\circ} \mathrm{C}\right)$ por um período de até sete dias, ou mais se necessário, para retirar a semente do período de dormência. Desse modo, não pôde ser incluída nas análises de dados a seguir. Para as demais sementes, as porcentagens de germinação foram superiores a $70 \%$, indicando que todas as espécies proporcionaram dados confiáveis. Lapa (2014) apontou em seu estudo porcentagens de germinação de $97 \%$ para as sementes da espécie L. sativa e ressaltou que a confiabilidade do teste de fitotoxicidade com as espécies de hortaliças dependem de que a porcentagem de germinação seja superior a $65 \%$ do total de sementes usadas na amostragem (USEPA, 1989).

A Figura 2 apresenta os valores médios dos comprimentos das radículas $(\mathrm{mm})$ para os diferentes controles empregados.

As sementes da espécie E. sativa germinadas em 120 horas foram as que apresentaram maior crescimento radicular médio $(97,0 \pm 17,6) \mathrm{mm}$, seguida pelas sementes de $L$. sativum $(88,5 \pm 6,3) \mathrm{mm}$ germinadas em 96 horas, ambas no controle positivo e com a porcentagem de germinação absoluta (\% GA) igual a $100 \%$ de todas as sementes.

Diferentemente do estudo de Lapa (2014), a espécie L. sativa apresentou os menores valores de crescimento radicular médio para todos os controles empregados, de $(12,8 \pm 3,1) \mathrm{mm}$ para o controle positivo, $(9,0 \pm 1,8) \mathrm{mm}$ para o controle neutro e $(4,3 \pm 0,8)$ $\mathrm{mm}$ para o controle negativo, assim como a menor porcentagem de germinação absoluta 
(\%GA), onde as sementes germinadas na água ISO, na água destilada e no dicromato de potássio resultaram em $70 \%, 83 \%$ e $85 \%$, respectivamente.

Figura 2: Valores médios dos comprimentos das radículas $(\mathrm{mm})$ e desvio padrão para todos os cultivos empregados.

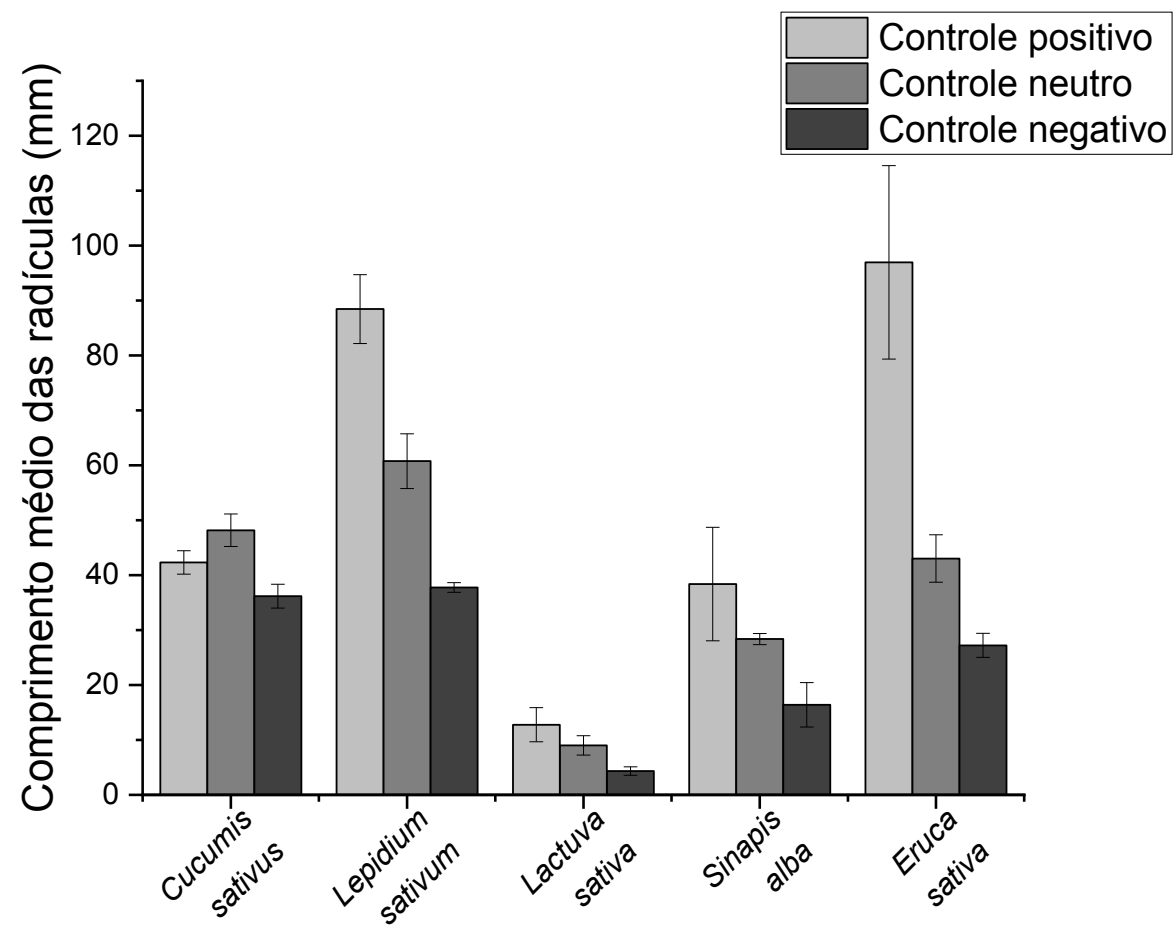

Espécie das sementes

Justifica-se o resultado do teste de fitotoxicidade da espécie $L$. sativa pela ausência dos tratamentos propostos pelas RAS (BRASIL, 2009), como pré-esfriamento à baixa temperatura $\left(10^{\circ} \mathrm{C}\right)$ por três dias e desejável uso de luz antes da realização do teste para as sementes dormentes.

As sementes da espécie $C$. sativus germinaram em todos os controles com excelente taxa de germinação, superior a 97,5\%, e obteve maior crescimento radicular médio na água destilada $(28,2 \pm 1,5) \mathrm{mm}$, seguido pelo controle positivo $(48,2 \pm 2,9) \mathrm{mm}$ e com menor valor no controle negativo $(36,16 \pm 2,2) \mathrm{mm}$. Cruz et al. (2013), estudaram as sementes da espécie $C$. sativus e concluíram que o seu alongamento radicular foi o melhor parâmetro para avaliar a redução da toxicidade dos compostos analisados (óleo diesel e óleo lubrificante).

As sementes da espécie E. sativa apresentaram crescimento expressivo e Índice de Germinação (IG) similar ao da espécie L. sativum com estimulação ao crescimento na água ISO e efeito fitotóxico com o dicromato de potássio (Tabela 4). A classificação do Índice de Germinação (IG), de Lumbaque et al. (2016), se mostrou menos abrangente quanto a classificação de Belo (2011), em que apresentou resultados "não fitotóxico", "fitotóxico", "moderadamente fitotóxico" e "potencialização na germinação" para as espécies das sementes analisadas. 
Tabela 4. Índice de Germinação (IG) calculado para cada espécie de semente e classificação quanto ao nível de fitotoxicidade.

\begin{tabular}{|c|c|c|c|c|}
\hline ESPÉCIE & CONTROLE & IG & $\begin{array}{l}\text { CLASSIFICAÇÃO } \\
\text { (Belo, 2011) }\end{array}$ & $\begin{array}{c}\text { CLASSIFICAÇÃO } \\
\text { (Lumbaque et al., 2016) }\end{array}$ \\
\hline \multirow[b]{2}{*}{ Cucumis sativus } & Positivo & 90,09 & \multirow{2}{*}{$\begin{array}{c}\text { Não fitotóxico } \\
\text { Moderadamente } \\
\text { fitotóxico }\end{array}$} & \multirow{2}{*}{$\begin{array}{l}\text { Estímulo } \\
\text { Nenhum Efeito } \\
\text { Significativo }\end{array}$} \\
\hline & Negativo & 76,97 & & \\
\hline \multirow{2}{*}{ Lepidium sativum } & Positivo & 145,58 & \multirow{2}{*}{$\begin{array}{l}\text { Potencializa a } \\
\text { germinação } \\
\text { Moderadamente } \\
\text { fitotóxico } \\
\end{array}$} & Estímulo \\
\hline & Negativo & 62,14 & & Inibição \\
\hline \multirow{2}{*}{ Lactuca sativa } & Positivo & 120,23 & \multirow{2}{*}{$\begin{array}{l}\text { Potencializa a } \\
\text { germinação } \\
\text { Fitotóxico }\end{array}$} & Estímulo \\
\hline & Negativo & 49,63 & & Inibição \\
\hline \multirow{2}{*}{ Sinapis alba } & Positivo & 128,55 & \multirow{2}{*}{$\begin{array}{l}\text { Potencializa a } \\
\text { germinação } \\
\text { Fitotóxico }\end{array}$} & Estímulo \\
\hline & Negativo & 54,89 & & Inibição \\
\hline \multirow{2}{*}{ Eruca sativa } & Positivo & 225,31 & \multirow{2}{*}{$\begin{array}{l}\text { Potencializa a } \\
\text { germinação } \\
\text { Moderadamente } \\
\text { fitotóxico }\end{array}$} & Estímulo \\
\hline & Negativo & 63,26 & & Inibição \\
\hline
\end{tabular}

As sementes da espécie $S$. alba e $L$. sativa se mostraram sensíveis ao controle negativo, apresentando o IG "fitotóxico" e não apresentando nenhum tipo de alteração na estrutura das raízes, apenas inibição em seu crescimento radicular médio e a classificação "potencializa a germinação" para o controle positivo. As sementes da espécie C. sativus, apesar de também não apresentarem nenhum dano à sua estrutura radicular, resultou em "moderadamente fitotóxico" para o controle negativo e "não fitotóxico" para o controle positivo. Essa diferença na sensibilidade das sementes e alteração na classificação do IG é explicado por Cruz et al. (2013) e Lapa (2014), em que o tamanho da semente influencia diretamente na sensibilidade da toxicidade, onde a semente de maior tamanho oferece maior proteção quando exposta ao contaminante. Tal afirmação é coerente quanto à semente da espécie $C$. sativus, devido ao seu tamanho avantajado. Entretanto, a semente da espécie L. sativa apesar do seu tamanho reduzido, apresentou menor crescimento médio das radículas quando comparado às demais sementes pequenas, como as da espécie E. sativa, por exemplo. Pode-se explicar este resultado pela ausência do tratamento indicado pelas RAS (BRASIL, 2009) para a espécie $L$. sativa, em que se recomenda pré-esfriar as sementes a $10^{\circ} \mathrm{C}$ por três dias e fornecer luz adicional para despertar as sementes dormentes (BRASIL, 2009).

Para as espécies de $L$. sativum e $E$. sativa, foram observados danos à estrutura das radículas que estiveram em contato com o controle negativo, tais como: crescimento tortuoso, espessura fina e partes acinzentadas, dificultando a determinação do seu crescimento radicular. Diversos mecanismos estão envolvidos na inibição do crescimento de raízes. Como exemplo, Macan et al. (2012) sugerem que o efeito fitotóxico está na capacidade do composto de se acumular e interagir em sua parede celular, promovendo consequências que desencadeiam alterações fisiológicas, bioquímicas e moleculares, sendo elas: alteração do fluxo de água e nutrientes, alteração da homeostase do fluxo de 
cálcio, distúrbios do ciclo celular, entre outros, justificando assim, a aparência tortuosa das radículas e partes escuras.

Comparando estatisticamente as espécies das sementes, o resultado do teste de normalidade mostrou que os dados provêm de uma distribuição normal, possibilitando o uso da ANOVA de fator único. Para os dados de IG, a ANOVA para os dados do controle positivo, resultou em $p=7,0210^{-9}$ e para o controle negativo, $p=1,7110^{-10}$, indicando que pelo menos dois grupos de espécies tinham significância estatística. $\mathrm{Na}$ Tabela 5 são apresentados os resultados do Teste de Tukey para as duplas de controles (positivo e negativo) para cada espécie de semente. Nessa tabela, também é possível visualizar que os resultados dos bioensaios com sementes das espécies $C$. sativus, L. sativa e $S$. alba, são estatisticamente iguais para o controle positivo. Os resultados dos bioensaios com sementes da espécie de $L$. sativum e $L$. sativa, assim como os das sementes das espécies $S$. alba e E. sativa são estatisticamente iguais no controle negativo.

Tabela 5: Análise de variância da mesma semente para cada conjunto de controle empregado. Nota: letras diferentes indicam diferença estatística pelo teste de Tukey $(p<0,05)$.

\begin{tabular}{ccc}
\hline ESPÉCIE & IG (CONTROLE POSITIVO) & $\begin{array}{c}\text { IG (CONTROLE } \\
\text { NEGATIVO) }\end{array}$ \\
\hline Cucumis sativus & $87,18 \pm 2,17^{\mathrm{b}}$ & $80,42 \pm 2,22^{\mathrm{c}}$ \\
Lepidium sativum & $136,37 \pm 6,26^{\mathrm{a}}$ & $55,70 \pm 0,87^{\mathrm{a}}$ \\
Lactuca sativa & $78,71 \pm 4,35^{\mathrm{b}}$ & $48,69 \pm 1,86^{\mathrm{a}}$ \\
Sinapis alba & $86,53 \pm 11,31^{\mathrm{b}}$ & $60,29 \pm 5,13^{\mathrm{b}}$ \\
Eruca sativa & $193,03 \pm 17,60^{\mathrm{c}}$ & $60,77 \pm 2,17^{\mathrm{b}}$ \\
\hline
\end{tabular}

A semente da espécie $S$. alba possui uso na culinária e grande facilidade em ser adquiridas sem tratamento no comércio, enquanto que as demais sementes, são encontradas em lojas de jardinagem e especializadas na agricultura, sendo normalmente peletizadas e tratadas com agrotóxicos para melhor desempenho no plantio. Isso as torna inviáveis no estudo da sensibilidade de elementos tóxicos no teste de germinação. Pela necessidade do estudo, solicitou-se junto à empresa de produção e comercialização de sementes $\left(\right.$ Isla $^{\circledR}$ ) que as sementes das espécies de $A$. cepa, C. sativus, $L$. sativum, $L$. sativa e $E$. sativa não obtivessem tratamento, o que demandou tempo de fabricação, embalagem e postagem, superior a 25 dias úteis para o recebimento das sementes e início dos testes. O comparativo econômico dos valores desembolsados para compra das sementes avalia o custo de aquisição para cada $50 \mathrm{~g}$ de semente. Os valores das sementes de $A$. cepa foi de $\mathrm{R} \$ 32,3 / 50 \mathrm{~g}$, para as sementes de $C$. sativus, $\mathrm{R} \$ 30,5 / 50 \mathrm{~g}$, as sementes da espécie de $L$. sativum, $R \$ 23,8 / 50 \mathrm{~g}$, L. sativa, $R \$ 20,5 / 50 \mathrm{~g}$, S. alba e $E$. sativa, $R \$ 1,25 / 50 \mathrm{~g}, \mathrm{R} \$ 11,3 / 50 \mathrm{~g}$, respectivamente. Destacando-se que as sementes adquiridas da espécie $S$. alba tiveram custo médio, cerca de $85 \%$ mais baixo que as demais.

\section{CONCLUSÕES}

Conclui-se que a semente da espécie $S$. alba obteve o melhor desempenho no teste de fitotoxicidade, por apresentar menor valor de compra, alta germinação em menor tempo de incubação, facilidade de avaliação das plântulas e elevado grau de sensibilidade frente aos compostos estimulante e inibidor. 


\section{REFERÊNCIAS}

ABNT. Associação Brasileira de Normas Técnicas. NBR 15088: Ecotoxicologia aquática - Toxicidade aguda - Método de ensaio com peixes (Cyprinidae). Rio de Janeiro: ABNT, 2016.

BEDELL, J.P.; FERRO, Y.; BAZIN, C.; PERRODIN, Y. (a) Selection of a halophytic plant for assessing the phytotoxicity of dredged seaport sediment stored on land.

Environmental Monitoring and Assessment, v. 186, p. 183-194, 2014.

BEDELL, J.P.; FERRO, Y.; BAZIN, C.; PERRODIN, Y. (b) Evaluation of phytotoxicity of seaport sediments aged artificially by rotary leaching in the framework of a quarry deposit scenario. Marine Pollution Bulletin, v. 86, p. 4858, 2014.

BELO, S.R.S. Avaliação de fitotoxicidade através de Lepidium sativum no âmbito de processos de compostagem. 68 f. Dissertação (Mestrado em Engenharia do Ambiente) - Universidade de Coimbra, Coimbra, Portugal, 2011.

BRASIL. Conselho Nacional do Meio Ambiente - CONAMA. Resolução CONAMA n. 344 de 2004. Dispõe sobre diretrizes gerais e os procedimentos mínimos para a avaliação do material a ser dragado em águas jurisdicionais brasileiras, e dá outras providências. Disponível em:

<http://www2.mma.gov.br/port/conama/legislacao/CONAMA_RES_CONS_2004_344.pdf> . Acesso em: 01 mar. 2019.

BRASIL. Conselho Nacional do Meio Ambiente - CONAMA. Resolução CONAMA n. 357 de 2005. Dispõe sobre a classificação dos corpos de água e diretrizes ambientais para o seu enquadramento, bem como estabelece as condições e padrões de lançamento de efluentes, e dá outras providências. Disponível em:

<http://www2.mma.gov.br/port/conama/res/res05/res35705.pdf>. Acesso em: 02 mar. 2019.

BRASIL. Ministério da Agricultura, Pecuária e Abastecimento. Regras para Análise de Sementes (RAS). Brasília: MAPA/Secretaria de Defesa Agropecuária, 2009. 399 p.

CARNIATO, J.G.; GERALDO, S.M.; PELEGRINI, N.N. de B.; PATERNIANI, J.E.S.; PELEGRINI, R.T. Avaliação da toxicidade de percolado de resíduos sólidos pós tratamento biológico e fotocatalítico. Engenharia Ambiental, v.4, n.2, p. 92-101, 2007.

CETESB. Companhia Ambiental do Estado de São Paulo. Ensaios ecotoxicológicos com organismos aquáticos: Atendimento à legislação Ambiental - Orientações para realização de ensaios e apresentação dos resultados nos documentos encaminhados à CETESB. ELHE - Setor de Ecotoxicologia Aquática. 2017.

COSTA, C.H. Estudo Ecotoxicológico para valorização do resíduo produzido no processo de polimento de piso porcelanato na indústria cerâmica. $153 \mathrm{f}$. Dissertação (Mestrado em Engenharia Ambiental) - Universidade Federal de Santa Catarina, Florianópolis, Santa Catariana, 2010. 
CRUZ, J.M.; LOPES, P.R.M.; MONTAGNOLLI, R.N.; TAMADA, I.S.; SILVA, N.M.M.G.; BIDOIA, E.D. Phytotoxicity of Soil Contaminated with Petroleum Derivatives and biodiesel. Ecotoxicology and Environmental Contamination, v. 8, n. 1, p. 49-54, 2013.

DEVESA-REY, R., MOLDES, A.B., DIÁZ-FIERROS, F., BARRAL, M.T. Toxicity of Anllóns River Sediment Extracts Using Microtox and the Zucconi Phytotoxicity Test. Bulletin of Environmental Contamination and Toxicology, v. 80, p. 225-230, 2008.

EMAMI, S.; POURBABAEI, A.A., ALIKHANI, H.A. Interactive effect of nitrogen fertilizer and hydrocarbon pollution on soil biological indicators. Environmental Earth Sciences, 2014.

FANTIN, A.C.M.; FREITAS, D.P.C.; FAGNANI, G.F.; LAPA, T.C.M.A. Teste de sensibilidade em sementes de Rúcula (Eruca sativa) e Alface (Lactuca sp.) em contato com diferentes concentrações do pesticida Glifosato. Limeira:

UNICAMP-CESET, 2009.

FRECCIA, B. Avaliação da toxicidade e fitotoxicidade das águas do Rio Urussanga antes e após o tratamento com sedimento gerados em estação de tratamento de efluente de mineração de carvão. Criciúma: Universidade Extremo Sul Catarinense-UNESC, 2011.

GUERRA, R.C.; ANGELIS, D.F. Classificação e fitotoxicidade do lodo gerado pelo tratamento da água de produção do petróleo no terminal almirante barroso (Tebar), São Sebastião, SP. Rio Claro: Universidade Estadual Paulista - UNESP, 2012.

HIMANEN, M.; PROCHAZKA, P.; HANNINEN, K.; OIKARI, A. Phytotoxicity of low-weight carboxylic acids. Chemosphere, v. 88, p. 426-431, 2012.

KOHATSU, M.Y.; JESUS, T.A.; COELHO, L.H.G.; PEIXOTO, D.C.; POCCIA, G.T.; HUNTER, C. Fitotoxicidade de água superficial da Região Metropolitana de São Paulo utilizando bioensaio com Sinapis alba. Acta Brasiliensis, v. 2, p. 58-62, 2018.

KUNDRÁT, J.T.; GYULAI, I.; SIMON, E.; MIZSEI, E.; BRAUN, M.; TÓTHMÉRÉSZ, B. Study of the effects of high levels of nutrients on Seed germination and root elongation. Polish Journal of Environmental Studies, v. 26, n. 4, p. 1585-1590, 2017.

LAPA, M.P. Avaliação Ecotoxicológica de Solos Impactados com Borra Oleosa Submetidos a Diferentes Tratamentos de Biorremediação. $62 \mathrm{f}$. Trabalho de Conclusão de Curso (Graduação em Engenharia Ambiental e Sanitária) - Universidade Federal de Pelotas, Pelotas, Rio Grande do Sul, 2014.

LUMBAQUE, E.C.; GOMES, F.M.; CARVALHO, V.S.; FREITAS, A.M.; TIBURTIUS, E.R.L. Degradation and ecotoxicity of dye Reactive Black 5 after reductive-oxidative process.

Environmental Science and Pollution Research, v. 24, p. 6126-34, 2016. 
MACAN, J.M.; TEIXEIRA, G.A.; PICH, C.T.; PEDROSA, R.C.; FÁVERE, V.T.; GEREMIAS, R. Avaliação da toxicidade de drenagem ácida de mina de carvão, utilizando parâmetros físico-químicos e bioensaios. Revista Brasileira de Biociências, v. 10, n. 3, p. 275-80, 2012.

MACENA, D.Â.; AGOSTINI, E.A.T.; COSTA, T.T.; AGOSTINI, R.T. Teste de fitotoxicidade do composto atrazina: bioensaio utilizando como organismo teste a Lactuca sativa. Colloquium Vitae, v. 9, n. especial, p. 7-13, 2017.

MACÍA, P.; FERNÁNDEZ-COSTAS, C.; RODRÍGUEZ, E.; SIEIRO, P.; PAZOS, M.; SANROMÁN, M. A., 2014. Technosols as a novel valorization strategy for an ecological management of dreadged marine sediments. Ecological Engineering, v. 67, p. 182-9, 2014.

MATSUBARA, M.E. Remoção do antibiótico amoxicilina por biorreator com membrana operado em regime de pré-desnitrificação: avaliação do desempenho, identificação de subprodutos e análises ecotoxicológicas. 155 f. Dissertação (Mestrado em Ciência e Tecnologia Ambiental) - Universidade Federal do ABC, Santo André, São Paulo, 2018.

OECD. Test n. 202: Daphnia sp. Acute Immobilisation Test, OECD Guidelines for the Testing of Chemicals, Section 2, OECD Publishing, Paris, 2004. Disponível em: <https://doi.org/10.1787/9789264069947-en>, acesso em: 10 de janeiro de 2019.

PELEGRINI, N.N.B.; PATERNIANI, J.E.S.; CARNIATO, J.G.; SILVA, N.B.E PELEGRINI, R.T. Estudo da sensibilidade de sementes de Eruca Sativa (Rúcula) utilizando substâncias tóxicas para agricultura. XXXV Congresso Brasileiro de Engenharia Agrícola (CONBEA), João Pessoa-PB, 2006.

ROCHA, B.S. Fitotoxicidade do corante laranja reativo 64 como efluente têxtil tratado por oxidação avançada (US/H2O2). $50 \mathrm{f}$. Trabalho de Conclusão de Curso (Graduação em Engenharia Química) - Universidade Federal do Rio Grande do Norte, Natal, Rio Grande do Norte, 2017.

RUBINGER, C.F. Seleção de métodos biológicos para avaliação toxicológica de efluentes industriais. $89 \mathrm{f}$. Dissertação (Mestrado em Saneamento, Meio Ambiente e Recursos Hídricos) - Universidade Federal de Minas Gerais, Belo Horizonte, Minas Gerais, 2009.

SILVA, J.N.; MATTIOLO, S.R. Fitotoxicidade do dicromato de potássio $\left(\mathrm{K}_{2} \mathrm{Cr}_{2} \mathrm{O}_{7}\right)$ em sementes de alface (Lactuca sativa). São Paulo: Centro Tecnológico da Marinha (CTMSP), 2011.

SONG, B.; ZENG, G.; GONG, J.; ZHANG, P.; DENG, J.; DENG, C.; YAN, J.; XU, P.; LAI, C.; ZHANG, C.; CHENG, M. Effect of multi-walled carbon nanotubes on phytotoxicity of sediments contaminated by phenanthrene and cádmium. Chemosphere, v. 172, p. 449-458, 2017. 
USEPA. United States Environmental Protection Agency. Protocols for short term toxicity screening of hazardous waste sites $-600 / 3-88 / 029$, Washington, DC, 1989.

YERUSHALMI L.; ROCHELEAU, S.; CIMPOIA, R.; SARRAZIN, M.; SUNAHARA, G.; PEISAJOVICH, A.; LECLAIR, G.; GUIOT, S. Enhanced Biodegradation of Petroleum Hydrocarbons in Contaminated Soil. Journal of Soil Contamination, v. 7, n. 1, p. 37-51, 2010.

ZHANG, H.; MA, G.; SUN, L.; HUIYING, L. I. Effect of alkaline material on phytotoxicity and bioavailability of $\mathrm{Cu}, \mathrm{Cd}, \mathrm{Pb}$ and $\mathrm{Zn}$ in stabilized sewage sludge. Environmental Technology, v. 39, n. 17, p. 2168-217, 2017.

Recebido em: 23/04/2019

Aprovado em: 12/09/2019 